\title{
X-Ray measurements of radiation hard monolithic CMOS sensors at Diamond Light Source
}

\author{
Maria Mironova* \\ University of Oxford \\ E-mail: maria.mironova@cern.ch
}

on behalf of the MALTA team: P. Allport ${ }^{b}$, I. Berdalovic ${ }^{c, d}$, D. Bortoletto ${ }^{a}$, C.Buttar ${ }^{e}$, R. Cardella ${ }^{c, f}$, V. Dao ${ }^{c}$, M. Dyndal ${ }^{c}$, P. Freeman ${ }^{b}$, L. Flores Sanz de Acedo ${ }^{c, e}$, L. Gonella $^{b}$, T. Kugathasan ${ }^{c}$, K. Metodiev $^{a}$, H. Pernegger ${ }^{c}$, F. Piro ${ }^{c}$, R. Plackett ${ }^{a}$, P. Riedler $^{c}$, A. Sharma ${ }^{a, c}$, E.J. Schioppa $^{c}$, I. Shipsey ${ }^{a}$, C. Solans Sanchez ${ }^{c}$, W. Snoeys ${ }^{c}$, H. Wennlöf ${ }^{b}$, D. Weatherill ${ }^{a}$, D. Wood ${ }^{a}$, S. Worm ${ }^{b}$

${ }^{a}$ University of Oxford (UK)

${ }^{b}$ University of Birmingham (UK)

${ }^{c} \mathrm{CERN}(\mathrm{CH})$

${ }^{d}$ University of Zagreb (HR)

${ }^{e}$ University of Glasgow (UK)

${ }^{f}$ University of Oslo $(\mathrm{NO})$

\begin{abstract}
This contribution outlines the results of investigations into the effects of radiation damage in the mini-MALTA depleted monolithic pixel sensor prototype using a micro-focus X-ray beam at Diamond Light Source. The in-pixel photon response was measured for three different pixel design variations: one with the standard continuous $\mathrm{n}^{-}$layer layout and standard front-end, and extra deep p-well and $\mathrm{n}^{-}$gap designs with a modified front-end. The standard design showed a decrease of $12 \%$ in pixel response after irradiation to $1 \mathrm{e} 15 \mathrm{n}_{\mathrm{eq}} / \mathrm{cm}^{2}$. The two new designs did not show a significant decrease in pixel response after irradiation.
\end{abstract}

The 28th International Workshop on Vertex Detectors - Vertex2019

13-18 October, 2019

Lopud, Croatia

${ }^{*}$ Speaker. 


\section{Introduction}

In light of the future upgrades for the High-Luminosity LHC, and for future HEP applications, there has been significant R\&D into monolithic sensors. First, the ALICE experiment developed a fully monolithic sensor for their new tracking system [1]. Based on its design, the MALTA chip was developed in the context of the ATLAS experiment, which has been studied in lab and testbeam measurements [2]. Most recently, the mini-MALTA prototype was developed, implementing design changes which are aimed at improving the pixel performance and achieving higher radiation hardness [3]. In the following, results of an X-Ray test-beam at Diamond Light Source are presented for the different designs and different levels of irradiation [4].

\section{The mini-MALTA prototype}

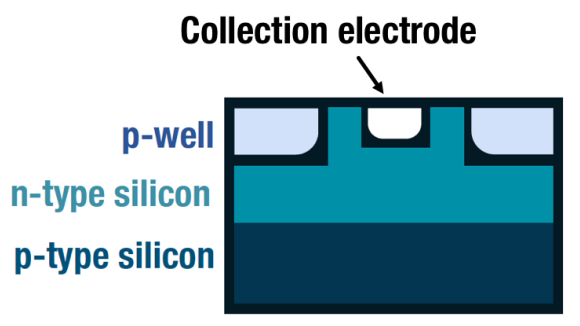

Continuous $\mathrm{n}^{-}$layer

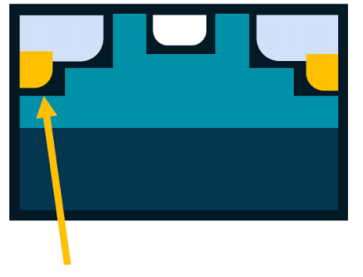

Extra-deep p-well

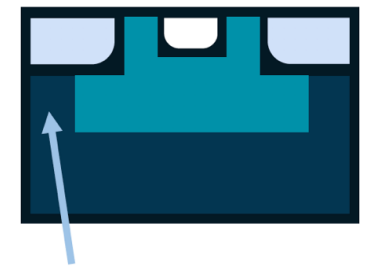

Gap in n- layer

Figure 1: Standard design and process modifications in mini-MALTA. The figure is not to scale, the thin n-type layer located on top of a p-type epitaxial layer with a depth of $30 \mu \mathrm{m}$. This layer is expected to be fully depleted, and is grown on a highly doped p-substrate which makes no contribution to the charge collection.

The mini-MALTA chip is a depleted monolithic pixel sensor prototype produced in $180 \mathrm{~nm}$ TowerJazz CMOS Image Sensor (CIS) technology. It has 64 x 16 pixels and 36.4 x $36.4 \mu \mathrm{m}^{2}$ pixel pitch, split into eight sectors which vary in their front-end design, reset mechanism and process. In particular, two process modifications have been introduced in mini-MALTA, based on the design of the MALTA chip. The standard design with a continuous $\mathrm{n}^{-}$layer, as well as the two process modifications, are shown in Figure 1. Both of these modifications are aimed at better charge collection at the pixel edges, by shaping the electric field lines such that the produced charge carriers are steered more directly towards the collection electrode in the centre of the pixel. The first design modification has an additional deep p-type implant and the second design has a gap in the $\mathrm{n}^{-}$layer. The the gap in the $\mathrm{n}^{-}$layer is only a mask modification, wheres the additional deep p-type implant is a process modification. Both of the modified designs have been previously shown to perform well in TCAD simulations [5].

All three designs are implemented with a standard and a modified front-end, where the latter has enlarged transistors to reduce Random Telegraph Signal (RTS) noise. For the standard continuous $\mathrm{n}^{-}$layer design, the sector with the standard front-end was tested, to be consistent with what was previously implemented in the MALTA chip, and for the new designs the sectors with enlarged transistors were measured. 


\section{Setup}

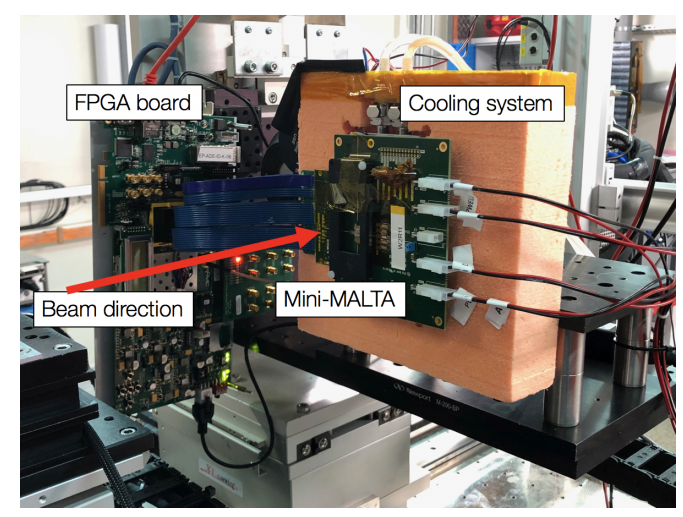

(a) Experimental Setup in the beamline.

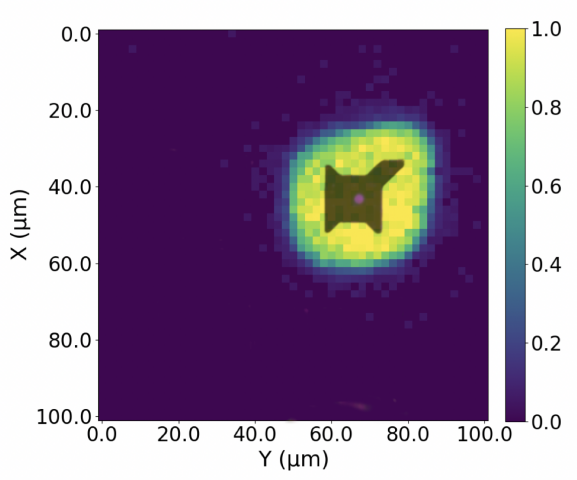

(b) Overlay of the single pixel map and the shape of the p-well cutout.

Figure 2: Picture of the experimental setup at the B16 beamline at Diamond Light Source and example of a single pixel map.

The experimental setup at the B16 beamline at Diamond Light Source is shown in Figure 2(a). The $8 \mathrm{keV}$ X-ray beam was focused using a Compound Reflective Lens (CRL) X-ray mirror arrangement and attenuated by $0.5 \mathrm{~mm}$ of aluminium. Using a knife edge technique the beam spot was measured to be $2 \mu \mathrm{m}$ (FWHM) [6]. The mini-MALTA PCB with the mounted chip was placed onto a motion stage and, for the irradiated samples, cooled down to $-13 \mathrm{C}$, using a cooling system based on a Peltier element and a water-cooled chuck. During the measurements, the mini-MALTA was moved in $2 \mu \mathrm{m}$ steps in both orthogonal directions to the beam. Data were acquired for $1 \mathrm{~s}$ at each position, using a readout system based on the Kintex KC705 FPGA board. Each of the scans covered an area of $100 \times 100 \mu \mathrm{m}^{2}$, which fully contained at least four pixels.

The properties of the tested samples are summarised in table 1. All three samples stem from the same wafer, thus they have the same resistivity and the same thickness of the epitaxial layer of $30 \mu \mathrm{m}$. The main interest of this study was to compare the pixel performance at different levels of irradiation. We tested one unirradiated device, one device irradiated up to $5 \mathrm{e} 14 \mathrm{n}_{\mathrm{eq}} / \mathrm{cm}^{2} \mathrm{using}$ $27 \mathrm{MeV}$ protons at the MC40 cyclotron at Birmingham [7], and one device irradiated to 1e15 $\mathrm{n}_{\mathrm{eq}} / \mathrm{cm}^{2}$ using neutrons (with an energy spectrum up to a few MeV) at the Ljubljana JSI TRIGA reactor [8]. The measurements for the different samples were performed at different thresholds, depending on the amount of noise in the chip. The threshold was set using the discriminator bias current ("IDB") and measured with a threshold scan for each of the sectors of each device, with the resultant thresholds shown in Table 1.

\section{Results}

\subsection{Single Pixel response}

In the analysis, the number of hits registered in a particular pixel was considered and plotted as a function of motion stage position. Then this number of hits was normalised to the number of 


\begin{tabular}{|l|l|l|l|l|l|}
\hline Sample & $\begin{array}{l}\text { Fluence } \\
\mathrm{n}_{\mathrm{eq}} / \mathrm{cm}^{2}\end{array}$ & $\begin{array}{l}\text { TID } \\
(\text { MRad })\end{array}$ & $\begin{array}{l}\text { continuous } \mathrm{n}^{-} \\
\text {layer threshold (e) }\end{array}$ & $\begin{array}{l}\text { Extra deep p-well } \\
\text { threshold (e) }\end{array}$ & $\begin{array}{l}\mathrm{n}^{-} \text {gap threshold } \\
(\mathrm{e})\end{array}$ \\
\hline \hline W2R11 & 0 & 0 & 368 & 197 & 190 \\
\hline W2R9 & $5 \mathrm{e} 14(\mathrm{p})$ & 66 & 533 & 303 & 274 \\
\hline W2R1 & $1 \mathrm{e} 15(\mathrm{n})$ & 1 & 330 & 181 & 187 \\
\hline \hline
\end{tabular}

Table 1: Table of mini-MALTA chips with their respective delivered dose in neutron equivalent fluence and Total Ionising Dose (TID), as well as the thresholds for the different samples and sectors.

\begin{tabular}{|l|l|l|l|l|}
\hline Sample & $\begin{array}{l}\text { Fluence } \\
\mathrm{n}_{\text {eq }} / \mathrm{cm}^{2}\end{array}$ & $\begin{array}{l}\text { continuous } \\
\mathrm{n}^{-} \text {response }(\%)\end{array}$ & $\begin{array}{l}\text { Extra deep p-well } \\
\text { response (\%) }\end{array}$ & $\mathrm{n}^{-}$gap response (\%) \\
\hline \hline W2R11 & 0 & $88.3 \pm 2.4$ & $90.5 \pm 2.2$ & $90.9 \pm 2.2$ \\
\hline W2R9 & $5 \mathrm{e} 14(\mathrm{p})$ & $81.2 \pm 2.8$ & $87.6 \pm 4.2$ & $88.4 \pm 3.8$ \\
\hline W2R1 & $1 \mathrm{e} 15(\mathrm{n})$ & $75.4 \pm 3.8$ & $90.5 \pm 2.8$ & $89.0 \pm 3.1$ \\
\hline \hline
\end{tabular}

Table 2: Calculated average pixel responses for the different samples and designs.

hits in the pixel center. An example of a single pixel map for the irradiated continuous $\mathrm{n}^{-}$layer design is shown in Figure 2(b). The pixel shape is clearly asymmetric. This is due to the shape of the p-well cutout, shown as an overlay in Figure 2(b), which is also asymmetric and extended in one direction. The larger cutout leads to a larger potential difference between collection electrode and p-well, thus the the charge collection is better where the p-well cutout is extended.

The same single pixel maps for the different designs and two different levels of irradiation are shown in Figure 3, along with the nominal pixel area of $36.4 \times 36.4 \mu \mathrm{m}^{2}$ as a white square. The asymmetric pixel shape is less visible in the two modified designs, as the charge collection there is dominated by the improvements from the design modifications.

To quantify the pixel performance, the pixel response was defined as the average of the normalised number of hits across the nominal pixel area. This value was calculated for each pixel individually and then averaged across all pixels which were fully visible in a scan. The response was calculated for the different samples and designs and the results are summarised in Table 2. The errors on the response values are calculated form the combination of the uncertainty on the normalisation and the pixel to pixel variation in response. For the continuous $\mathrm{n}^{-}$layer design a clear decrease of pixel response is observed with increasing irradiation, from $88 \%$ to $75 \%$. This is also clearly visible in Figures 3(a) and 3(d), which show a significant decrease in response at the pixel edges for the irradiated sample. In comparison, the two new designs do not show a decrease of response with irradiation. The charge collection shape becomes more symmetric, as the effects of radiation damage dominate. However, the size of the charge collection region remains the same and there is no significant decrease of pixel response around the pixel edges. Both of the new designs, with the additional p-type implant and with the gap in the $\mathrm{n}^{-}$layer, show similar results.

\subsection{Charge sharing}

Figure 4 shows the pixel response maps for all of the pixels at the same time. In addition, 


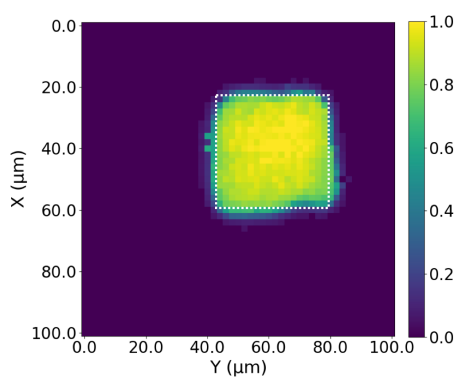

(a) W2R11 continuous $\mathrm{n}^{-}$layer.

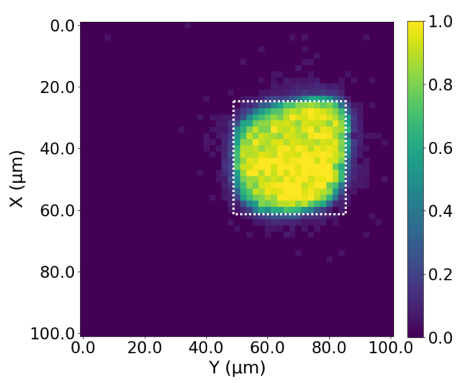

(d) W2R1 continuous $\mathrm{n}^{-}$layer.

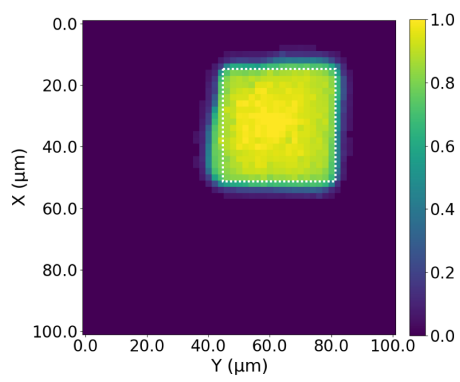

(b) W2R11 extra deep p-well.

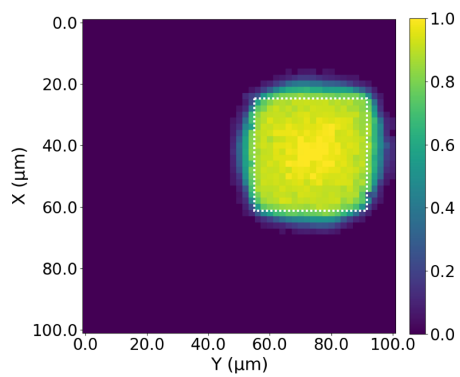

(e) W2R1 extra deep p-well.

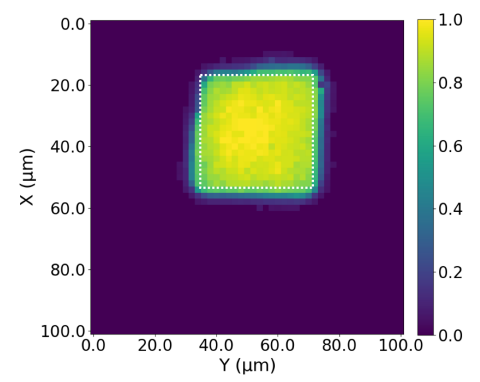

(c) W2R11 $\mathrm{n}^{-}$gap.

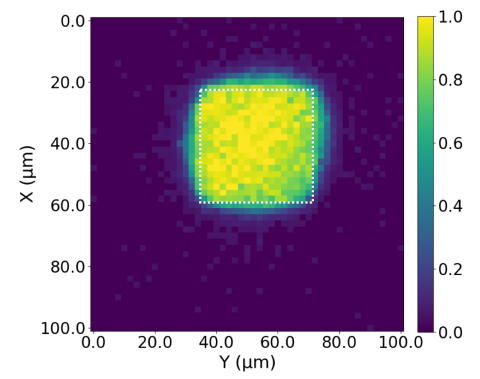

(f) $\mathrm{W} 2 \mathrm{R} 1 \mathrm{n}^{-}$gap.

Figure 3: Response maps for the different samples. For each sector the continuous $\mathrm{n}^{-}$layer, extra deep $\mathrm{p}$-well and $\mathrm{n}^{-}$gap sector are shown. W2R11 is unirradiated and W2R1 is neutron-irradiated to $1 \mathrm{e} 15 \mathrm{n} / \mathrm{cm}^{2}$.

the bottom panel in each plot shows the profile of the response at a particular $x$ location, both for the individual pixels and for the summed response. In these plots, there are regions where the summed response has values above one, which is where the charge is collected by two pixels at the same time, i.e. charge sharing occurs. Notably, for the continuous $\mathrm{n}^{-}$layer design on the unirradiated device, some of the corners already show a response below $100 \%$. For the new designs all of the corners are fully responsive. This is caused by the fact that the standard design was tested using the standard front-end and the new designs were tested for the modified front-end, which means that for the latter the threshold was lower, as shown in Table 1. Additionally, the process modifications strongly accelerate the signal collection in the new designs, thus the signal in the corners is recovered. After irradiation, the charge sharing for the standard design decreases significantly, in agreement with the loss of response in the pixel corners. For the new designs, however, the charge sharing regions seem to get broader with irradiation.

\section{Conclusions}

In the X-Ray test-beam at Diamond Light Source the performance of the mini-MALTA depleted monolithic pixel sensor prototype was tested for different levels of irradiation. The original design with a continuous $\mathrm{n}^{-}$layer shows a significant decrease in pixel response after irradiation to $1 \mathrm{e} 15 \mathrm{n}$ eq $/ \mathrm{cm}^{2}$. The two design modifications, with an additional p-type implant or a gap in the $\mathrm{n}^{-}$layer, improve the charge collection at the pixel edges and show no loss in pixel response after 


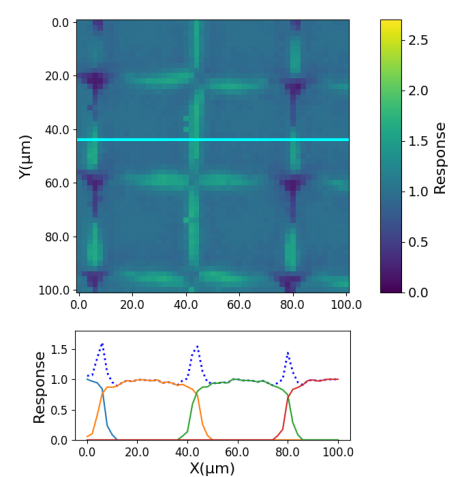

(a) W2R11 continuous $\mathrm{n}^{-}$layer.

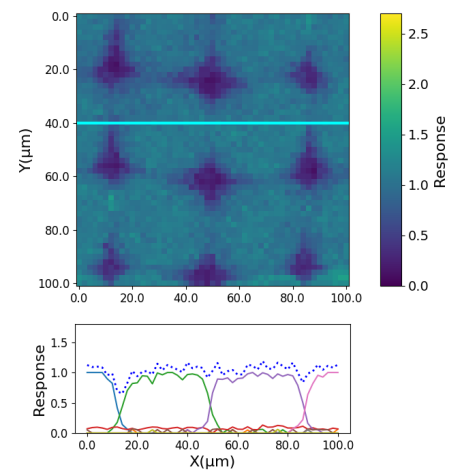

(d) W2R1 continuous $\mathrm{n}^{-}$layer.

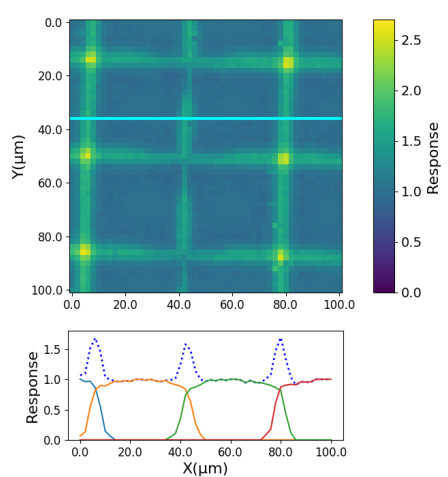

(b) W2R11 extra deep p-well.
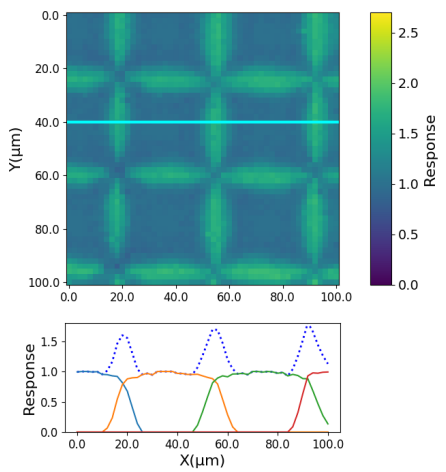

(e) W2R1 extra deep p-well.
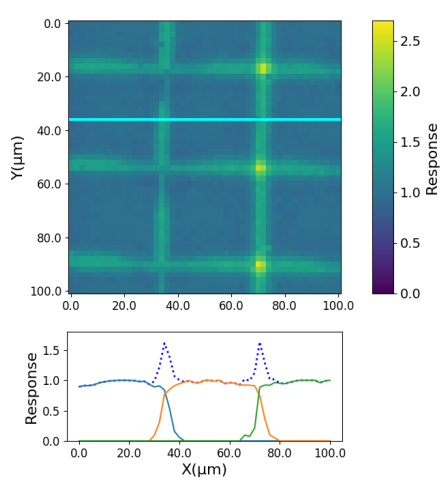

(c) W2R11 $\mathrm{n}^{-}$gap.
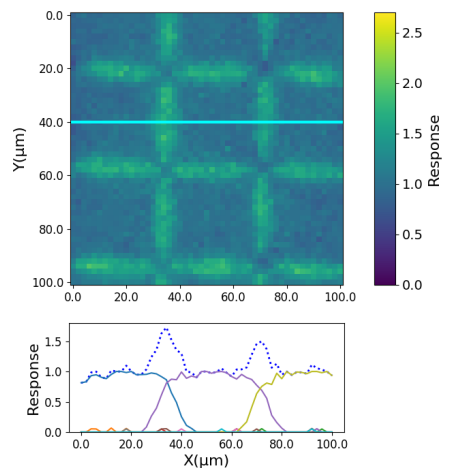

(f) $\mathrm{W} 2 \mathrm{R} 1 \mathrm{n}^{-}$gap.

Figure 4: Map of response for the different sectors in the W2R11 (unirradiated) and W2R1 (neutronirradiated to $1 \mathrm{e} 15 \mathrm{n}_{\mathrm{eq}} / \mathrm{cm}^{2}$ ) sample. The bottom panel shows the profile of response for a particular $\mathrm{x}$ location, which is indicated by a blue line.

receiving the same amount of irradiation. The two designs will be implemented in the next iteration of the chip and will undergo further testing.

\section{Acknowledgements}

This project has received funding from the European Union's Horizon 2020 Research and Innovation programme under Grant Agreement no. 654168. (IJS, Ljubljana, Slovenia). This research project has been supported by the Marie Sklodowska-Curie Innovative Training Network of the European Commission Horizon 2020 Programme under contract number 675587 "STREAM". We acknowledge Dr. Ben Phoenix, Prof. David Parker, Amelia Hunter, and the operators at the MC40 cyclotron in Birmingham (UK). We acknowledge Diamond Light Source for time on Beamline B16 under Proposal MM2206-1. 


\section{References}

[1] W. Snoeys et al. A process modification for CMOS monolithic active pixel sensors for enhanced depletion, timing performance and radiation tolerance. Nucl. Instrum. Methods Phys. Res. A, 871:90 96, 2017.

[2] E. J. Schioppa et al. Measurement results of the MALTA monolithic pixel detector. Nucl. Instrum. Methods Phys. Res. A, page 162404, 2019.

[3] M. Dyndal et al. Mini-MALTA: Radiation hard pixel designs for small-electrode monolithic CMOS sensors for the High Luminosity LHC. arXiv:1909.11987, Submitted to JINST.

[4] M. Mironova et al. Measurement of the relative response of TowerJazz Mini-MALTA CMOS prototypes at Diamond Light Source. arXiv:1909.08392, Submitted to Nucl. Instrum. Methods Phys. Res. A, 2019.

[5] M. Munker et al. Simulations of CMOS pixel sensors with a small collection electrode, improved for a faster charge collection and increased radiation tolerance. JINST, 14(05):C05013, May 2019.

[6] W. Yun et al. Nanometer focusing of hard x rays by phase zone plates. Review of Scientific Instruments, 70(5):2238-2241, 1999.

[7] P. Allport et al. Recent results and experience with the Birmingham MC40 irradiation facility. JINST, 12(03), 2017.

[8] L. Snoj, G. Žerovnik, and A. Trkov. Computational analysis of irradiation facilities at the JSI TRIGA reactor. Applied Radiation and Isotopes, 70(3):483 - 488, 2012. 